\title{
Preventing the introduction of SARS-CoV-2 into school settings
}

\author{
Nisha Thampi MD MSc, Beate Sander PhD, Michelle Science MD MSc
}

— Cite as: CMAJ 2021 January 4;193:E24-5. doi: 10.1503/cmaj.202568; early-released November 24, 2020

See related research at www.cmaj.ca/lookup/doi/10.1503/cmaj.202065

$\mathbf{E}$ nhanced health and safety measures have been introduced into schools and group activities around the world to prevent the transmission of severe acute respiratory syndrome coronavirus 2 (SARS-CoV-2) among students and staff. This includes screening for symptoms to prevent those who are symptomatic from entering schools. However, with the large variation in clinical manifestations of SARS-CoV-2 in children, schools have been presented with a particular challenge to identify those with SARS-CoV-2 infection before they enter the building.

Knowing which symptoms are more likely to be associated with SARS-CoV-2 infection may help to direct screening and testing criteria for children. In this context, a related study offers important insights. ${ }^{1}$ King and colleagues sought to determine the symptoms most likely to predict SARS-CoV-2 infection in a cohort of Albertan children and adolescents under 19 years of age. They found that $35.9 \%$ of children who received a first SARS-CoV-2 test between April and September 2020 and tested positive reported no symptoms, a higher proportion than the pooled estimates of $15 \%-20 \%$ from systematic reviews on pediatric coronavirus disease 2019 (COVID-19). ${ }^{2}$ This discrepancy may be due to the broad strategy of testing close contacts that has existed in Alberta since early April, including testing contacts who are asymptomatic or presymptomatic at specified times after exposure.

The related study also confirms the ubiquitousness of symptoms potentially related to COVID-19 among children and adolescents. Fever, cough and rhinorrhea were the most common symptoms among children tested for SARS-CoV-2 infection, regardless of whether they tested positive or negative. However, the symptoms most likely to be associated with a positive SARS-CoV-2 test result were anosmia/ageusia, nausea/vomiting, headache and fever/chills. A recent umbrella review identified more than 30 systematic reviews regarding the clinical manifestations of pediatric COVID-19. ${ }^{2}$ Predominant symptoms included fever and cough in more than half the cases, followed by rhinorrhea/nasal congestion, myalgia/fatigue and sore throat in $10 \%-20 \%$ of cases and gastrointestinal symptoms and headache in fewer than $10 \%$ of cases. ${ }^{2}$ Although anosmia, with or without ageusia, has emerged as a specific symptom of SARS-CoV-2 infection in nearly half of all adult cases, King and colleagues found these symptoms in only

\section{KEY POINTS}

- Children with severe acute respiratory syndome coronavirus 2 (SARS-CoV-2) infection are most likely to be asymptomatic, or to present with mild to moderate symptoms.

- Some symptoms, such as cough, rhinorrhea and fever, can be indistinguishable from those associated with other viral respiratory illnesses.

- Anosmia/ageusia has been shown to be strongly associated with a positive test for SARS-CoV-2 infection, but the true predictive value of many symptoms remains unknown.

- Since no symptom screening strategy will prevent every child with SARS-CoV-2 infection from entering school, additional schoolbased health and safety measures - including physical distancing, hand hygiene, masking, improved ventilation and outdoor learning opportunities - must continue to play a prominent role in preventing the spread of infection in this setting.

- Further studies are needed to understand secondary SARS-CoV-2 transmission among children and their households, and the conditions under which transmission occurs.

$7.7 \%$ of children who tested positive. ${ }^{1}$ This frequency is comparable to findings in a cohort of children of health care workers in the United Kingdom, but lower than the 20\% reported in a hospital-based pediatric cohort in Massachusetts. ${ }^{3-5}$

King and colleagues suggest that screening questionnaires should perhaps include only symptoms associated with positive test results, but they acknowledge the infrequency with which these symptoms occur, relative to upper respiratory tract symptoms and fever. Although the authors calculated positive likelihood ratios for the presence of each symptom in their analysis, this approach links the symptom to the infection but does not show whether the symptom occurred in isolation of other symptoms (e.g., Does isolated rhinorrhea, with or without an exposure history, predict SARS-CoV-2 infection?) or whether a child with rhinorrhea is likely to test positive for SARS-CoV-2. Given that symptoms such as headaches and rhinorrhea are common among school-aged children even in the absence of an infection, it would be important to determine the positive predictive value of these symptoms for COVID-19, particularly as isolated symptoms. 
For which symptoms, then, should children be screened before entering school? There are trade-offs between taking a broad versus narrow approach to symptom screening. Attempting to identify all students who may have symptomatic SARS-CoV-2 before entering the school environment would also result in the exclusion of many students with symptoms potentially unrelated to, yet indistinguishable from, COVID-19. Furthermore, the requirement for testing and isolation based on screening for a broad range of symptoms may rapidly overwhelm testing capacity and decrease the willingness of children and parents to report symptoms. Conversely, targeting specific but infrequent symptoms may result in children with unrecognized COVID-19 entering the school environment.

Given the high proportion of children with SARS-CoV-2 who remain asymptomatic, it is unlikely that any symptom screening strategy will prevent every child with SARS-CoV-2 infection from entering school. Therefore, school-based health and safety measures beyond screening - including physical distancing, hand hygiene, masking, improved ventilation and outdoor learning opportunities - play an essential role in preventing the spread of infection in this setting. ${ }^{6-8}$ It is important to gather information on secondary transmission within schools to evaluate these health and safety measures and adjust as necessary.

In addition to robust public health measures in testing, contact tracing and isolation of cases and their exposed contacts, noninvasive and rapid test result turnaround could help to identify children with mild or asymptomatic infections, and could result in greater compliance with testing and isolation recommendations. Network epidemiology, accompanied by phylogenetic analyses of case clusters, could be additional tools to understand SARS-CoV-2 transmission among children and their households, and the conditions under which transmission occurs (i.e., the duration, frequency and intensity of exposures), particularly among those who are asymptomatic carriers. ${ }^{9}$ Although children have been shown to have higher viral loads early in the course of illness, it would be important to correlate these laboratory findings with the risk of transmission over the course of illness, and against the effect of mitigation measures across age groups (preschool, elementary and high school). ${ }^{4,10}$

The clinical presentation of pediatric SARS-CoV-2 infection is often indistinguishable from other respiratory viral infections, yet the implications for school attendance are far greater. To better direct finite resources to public health and school-based measures, it is critical that transmission risks be understood and mitigated as much as possible, weighed against the known benefits of in-person learning. ${ }^{11}$

\section{References}

1. King JA, Whitten TA, Bakal JA, et al. Symptoms associated with a positive result for a swab for SARS-CoV-2 infection among children in Alberta, Canada: an observational study. CMAJ 2020 Nov. 24 [Epub ahead of print]. doi: 10.1503/cmaj.202568.
2. Ontario Agency for Health Protection and Promotion (Public Health Ontario). Coronavirus disease 2019 and the pediatric population: an umbrella review. Toronto: Queen's Printer for Ontario; 2020. Available: www.publichealthontario.ca/-/ media/documents/ncov/ipac/2020/10/covid-19-pediatric-population-umbrella -review-synthesis.pdf?la=en (accessed 2020 Nov. 10).

3. Ibekwe TS, Fasunla AJ, Orimadegun AE. Systematic review and meta-analysis of smell and taste disorders in COVID-19. OTO Open 2020;4: $2473974 X 20957975$.

4. Yonker LM, Neilan AM, Bartsch Y, et al. Pediatric severe acute respiratory syndrome coronavirus 2 (SARS-CoV-2): clinical presentation, infectivity, and immune responses. J Pediatr 2020 Aug. 20 [Epub ahead of print]. doi: 10.1016/j.jpeds.2020.08.037.

5. Waterfield T, Watson C, Moore R, et al. Seroprevalence of SARS-CoV-2 antibodies in children: a prospective multicentre cohort study. medRxiv 2020 Sept. 2 [preprint]. doi: 10.1101/2020.08.31.20183095.

6. Heavey L, Casey G, Kelly C, et al. No evidence of secondary transmission of COVID-19 from children attending school in Ireland, 2020. Euro Surveill 2020; 25:2000903.

7. Macartney K, Quinn HE, Pillsbury AJ, et al. NSW COVID-19 Schools Study Team. Transmission of SARS-CoV-2 in Australian educational settings: a prospective cohort study. Lancet Child Adolesc Health 2020;4:807-16.

8. Tupper P, Colijn C. COVID-19's unfortunate events in schools: mitigating classroom clusters in the context of variable transmission. medRxiv 2020 Oct. 22 [preprint]. doi: 10.1101/2020.10.20.20216267.

9. Lemieux J, Siddle KJ, Shaw BM, et al. Phylogenetic analysis of SARS-CoV-2 in the Boston area highlights the role of recurrent importation and superspreading events. medRxiv 2020 Aug. 25 [preprint]. doi: 10.1101/2020.08.23.20178236.

10. Heald-Sargent T, Muller WJ, Zheng X, et al. Age-related differences in nasopharyngeal severe acute respiratory syndrome coronavirus 2 (SARS-CoV-2) levels in patients with mild to moderate coronavirus disease 2019 (COVID-19). JAMA Pediatr 2020;174:902-3.

11. Christakis DA, Van Cleve W, Zimmerman FJ. Estimation of US children's educational attainment and years of life lost associated with primary school closures during the coronavirus disease 2019 pandemic. JAMA Netw Open 2020; 2:e2028786.

\section{Competing interests: None declared.}

This article was solicited and has not been peer reviewed.

Affiliations: Children's Hospital of Eastern Ontario (Thampi); Department of Pediatrics (Thampi), University of Ottawa, Ottawa, Ont.; Toronto Health Economics and Technology Assessment Collaborative (Sander), University Health Network; Institute of Health Policy, Management and Evaluation (Sander), University of Toronto; The Hospital for Sick Children (Science); Department of Pediatrics (Science), University of Toronto, Toronto, Ont.

Contributors: All authors contributed to the conception and design of the work, drafted the manuscript, revised it critically for important intellectual content, gave final approval of the version to be published and agreed to be accountable for all aspects of the work.

Content licence: This is an Open Access article distributed in accordance with the terms of the Creative Commons Attribution (CC BY-NC-ND 4.0) licence, which permits use, distribution and reproduction in any medium, provided that the original publication is properly cited, the use is non-commercial (i.e. research or educational use), and no modifications or adaptations are made. See: https://creativecommons.org/licenses/by-nc-nd/4.0/

Correspondence to: Nisha Thampi, nthampi@cheo.on.ca 